\title{
Assessing the validity of dual-minima algorithm for heel-strike and toe-off prediction for the amputee population
}

\author{
Zohaib Aftab, Email: dr.zohaib@ucp.edu.pk
}

August 27, 2021

\begin{abstract}
Assessment of gait deficits relies on accurate gait segmentation based on the key gait events of heel strike (HS) and toe-off (TO). Kinematicsbased estimation of gait events has shown promise in this regard especially using the leg velocity signal and gyroscopic sensors. However, its validation for the amputee population is not established in the literature. The goal of this study is to assess the accuracy of lower-leg angular velocity signal in determining the TO and HS instants for the amputee population. An open data set containing marker data of 10 subjects with unilateral transfemoral amputation during treadmill walking was used. A rule-based dual-minima algorithm was developed to detect the landmarks in the shank velocity signal indicating TO and HS events. The predictions were compared against the force platform data for 2595 walking cycles from 239 walking trials. Results showed considerable accuracy for the HS with a median error of $-1 \mathrm{~ms}$. The TO prediction error was larger with the median ranging from $35-84 \mathrm{~ms}$. The algorithm consistently predicted the TO earlier than the actual event. Significant differences were found between the prediction accuracy for the sound and prosthetic legs. The prediction accuracy was also affected by the subjects' mobility level (K-level) but was largely unaffected by gait speed. In conclusion, the leg velocity profile during walking can predict the heel-strike and toe-off events for the transfemoral amputee population with varying degrees of accuracy depending upon the leg side and amputee's functional ability level.
\end{abstract}

Keywords: Informatics; Gait segmentation; Gait analysis

\section{Introduction}

Gait analysis is a valuable tool in assessing various pathologies. Deviations from a healthy gait template often indicate underlying health conditions. For people with amputations, an accurate gait assessment leads to the development of more efficient prosthetic devices and better evaluation of clinical outcomes. That is why, more and more clinics are using technology-enabled gait analysis solutions as opposed to traditional visual gait analysis.

A pre-requisite to effective gait assessment is the estimation of key gait events of heel strike (HS) and toe-off (TO), which represent the moment the foot is placed and removed from the ground respectively [1]. 
Traditionally, these are estimated using the force or pressure data from specialized platforms in research laboratories. However, due to high cost and space constraints, this method is not used widely in clinics. Instead, kinematics-based solutions are becoming popular due to the possibility of body-worn inertial sensors. These methods rely on leg or foot velocity/acceleration data and rule-based algorithms to estimate gait events. Many authors have validated this approach for TO and HS detection for healthy subjects $[2,3,4,5,6,7,8,9,10,11]$ as well as for subjects with walking disorders $[12,13,14,15,16]$. However, its validity for the amputee population is not well established in the literature.

The kinematic methods require an algorithm to identify observable features in the velocity/acceleration data of body segments. Several rulebased algorithms have also been developed for this purpose. A popular choice is to use the shank angular velocity for TO and HS estimation corresponding to the minima in the sagittal-plane angular velocity signal [17]. Many researchers have exploited this signal over the years for diverse subject populations and reported a reasonable degree of accuracy $[18,19$, $7,3,20,14,21,22,2]$.

However, no study has focused on amputee subjects except [20] which included data from a single subject. Since lower-body amputations lead to gait deviations and compensatory movements, there is a need to evaluate this paradigm with a large data set of persons with amputations. Hence, the goal of this study is to compare the accuracy of gait event prediction using the shank angular velocity against force platform data. A secondary objective is to observe if this accuracy is affected by parameters such as leg side (sound or prosthetic), subjects' walking ability, and walking speed. A published data set of amputee subjects by Hood et. al. [23] is employed for this purpose containing optoelectronic and force plate data for transfemoral amputees.

\section{Materials and Methods}

A data set consisting of marker and force plate data of 18 individuals with unilateral transfemoral amputation was recently published [23]. It is the most comprehensive gait data set available for prosthesis users which provides force platform data for all steps taken during a trial. Moreover, the walking speed was controlled accurately on a treadmill as opposed to subjective instructions to walk 'slow' or 'fast'.

Subjects were divided into two groups based on their comfortable walking speed with the speed of $0.8 \mathrm{~m} / \mathrm{s}$ acting as a threshold. On the Medicare functional classification level (MFCL), subjects were either categorized as limited community ambulators (K-level 2) or full community ambulators (K-level 3) [24]. Each subject walked at five different speeds with K-level 2 subjects at $[0.4,0.5,0.6,0.7,0.8 \mathrm{~m} / \mathrm{s}]$, and K-level 3 subjects at $[0.6,0.8$, $1.0,1.2,1.4 \mathrm{~m} / \mathrm{s}]$. Complete details on the protocol and data acquisition are available in [23].

The original study contained an equal number of subjects in both groups. However, for this study, subjects using the handrails during walking were excluded to avoid the effect of secondary support on the gait pattern. This resulted in ten subjects for further analysis (including three K-level 2 and seven K-level 3 subjects). These subjects are listed in Table 1.

The data set reported four to five walking trials per speed resulting in 
Table 1: List of subjects for whom the walking data is used in this study. Complete details on amputation can be found in [23]

\begin{tabular}{lcccc}
\hline Subject & Age & Amputation side & K-level & Number of trials in the analysis \\
\hline \multicolumn{1}{l}{ K-level 2 } & subjects & & \\
\hline TF05 & 72 & Left & K2 & 25 \\
TF09 & 65 & Left & K2 & 25 \\
TF12** & 59 & Left & K2 & 23 \\
\hline K-level & subjects & & & \\
\hline TF01* & 26 & Right & K3 & 21 \\
TF07** & 49 & Left & K3 & 22 \\
TF08 & 42 & Right & K3 & 25 \\
TF11** & 51 & Right & K3 & 23 \\
TF16 & 36 & Left & K3 & 25 \\
TF17 & 38 & Left & K3 & 25 \\
TF19 & 30 & Left & K3 & 25 \\
\hline
\end{tabular}

a total of 246 walking trials for the ten subjects. However, after careful observation of the force platform and marker profiles, some trials were discarded due to either incomplete or erroneous data. This resulted in a total of 239 trials for the final analysis.

\section{Estimation of leg velocity signal from marker data}

The raw data consisted of three-dimensional trajectories of 61 cutaneous reflective markers. The data contained the .c3d files for the marker trajectories which were extracted using an open-source motion analyzer software MOKKA (Motion Kinematic and Kinetic Analyzer [25]). For each trial, the data were exported into a .csv file and read in Matlab for the calculation of lower-leg angular velocities from the coordinates of tibia markers. Two tibia markers on each leg (Figure 1) were used to estimate leg orientation using the method presented in [26]. The method uses two markers in line with the bone axis to calculate the orientation of the segment, which is further differentiated with respect to time to obtain the angular velocity.

The raw marker data was collected at $200 \mathrm{~Hz}$ and it is subject to a lot of noise due to soft tissue artifacts. To reduce the noise in the resulting angular velocity signal, a low pass filter was designed and implemented. For this purpose, the frequency spectrum and the Nyquist frequency of the signal for all subjects were analyzed. A cut-off frequency of $4 \mathrm{~Hz}$ gave was chosen which resulted in negligible loss of data and time-shift of the signal. An example of the filtering is shown in Figure 1 (right panel) for a trial at $1.2 m \cdot s^{-1}$.

\section{Algorithm}

The determination of TO and HS events in the velocity signal is based on the dual-minima approach similar to the one presented by [22]. It starts 


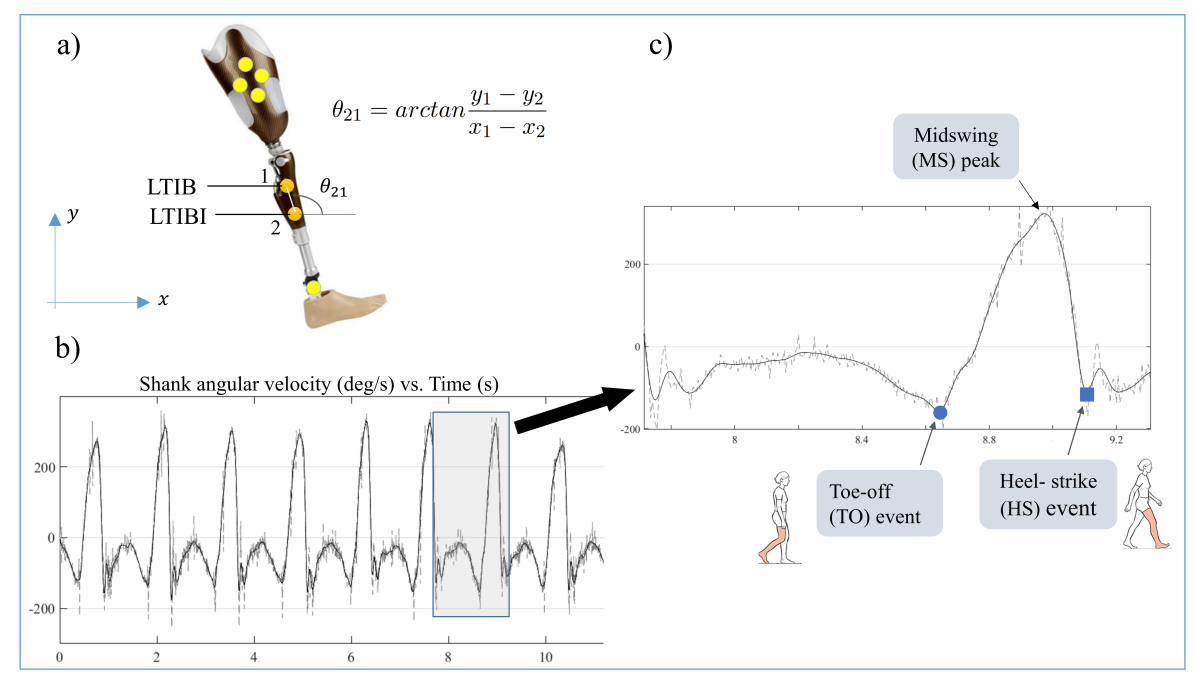

Figure 1: a): Placement of tibia markers for leg orientation and velocity calculation, b) A typical shank velocity signal with raw (grey) and filtered (black) data, c) Enlarged view of one gait cycle from the velocity signal. The algorithm starts with the detection of the largest positive peaks in the signal (marked as MS) which define the intervals for gait events. TO is identified as the last negative peak (or minima) just before the MS (circle) while HS is defined as the negative peak just after the MS (square)

with the detection of all the positive peaks of the signal. These positive peaks are associated with the midswing (MS) (c.f. Fig. 1). Each positive peak is accompanied by two negative peaks (or minima) on either side which indicate the reversal of leg velocity direction. The negative peak (NP) preceding the MS is identified as the toe-off event while the NP after the MS is marked as heel strike. The algorithm is implemented in Matlab.

\section{Statistical analysis}

For each walking cycle, the timings for the TO and HS events obtained by this algorithm are compared against the force platform-based timings provided in the data set. The errors (eTO, eHS) are calculated by taking the difference between the corresponding predicted and actual events.

$$
\operatorname{Error}(e H S / e T O)=t_{\text {actual }}-t_{\text {predicted }},
$$

where the actual events refer to the ones marked using the force platform. The error is positive when the predicted event precedes the actual event and vice versa.

Shapiro-Wilk test and visual inspection of histograms indicated that the error distribution was not normal. Hence a 5 number summary statistic (involving the median, lower and upper quartiles, minimum and maximum values) was selected for further descriptive analysis. Non-parametric statistical tests of significance were performed (at $\mathrm{p}=.05$ level) for group differences. 
Descriptive statistics of mean error (ME) and mean absolute error (MAE) were also computed to compare the results of this study with the literature which frequently report these averages.

\section{Results}

TO and HS events for a total of 2595 walking cycles from 239 trials were compared. Results are summarized in Figure 2. The median heel strike error (eHS) was $-1 \mathrm{~ms}$ with an interquartile range (IQR) of $31 \mathrm{~ms}$. The box extended both above and below the median, indicating the predictions to be early or late than the actual event. On the other hand, the median toe-off error (eTO), as well as the IQR, were larger (43ms and $51 \mathrm{~ms}$ respectively). The error is positive in all the cases indicating consistent early detection by the algorithm for TO. The mean absolute values shown at the bottom of the plot indicate that the magnitude of eHS was half as compared to eTO indicating a better HS estimation.

The accompanying violin plots (in grey) show the distribution of error values for both events. Interestingly, the distribution is bimodal in nature for the eTO with two local maxima. This indicates a dichotomy of results into two groups.

\section{Difference by the leg side}

To observe the differences in prediction error between legs (sound vs. prosthetic), separate error values are plotted for each leg in Figure 3. A Wilcoxon signed-rank test was also performed to reveal any significant differences.

For the heel strike, the median error remained closer for both legs (1ms and $-8 \mathrm{~ms}$ for sound vs. prosthetic side). But the error showed larger dispersion on the prosthetic side with an IQR of 53ms. For the TO event, there was clearly a large difference in the median error value between the two legs. The median error on the sound side was $84 \mathrm{~ms}$ versus $35 \mathrm{~ms}$ on the prosthetic side. This explains the bimodal distribution of error observed in Figure 2.

Statistically significant differences were found between the two sides for both TO and HS events $(p<.001)$.

\section{Effect of K-level and walking speed}

Finally, in order to observe the effect of subjects' functional classification level and walking speed on the prediction error, separate boxplots are produced for both subject groups at different walking speeds (Figure 4). Plots for trials from K2 subjects are shown on the left while trials from $\mathrm{K} 3$ subjects are on the right. Walking speed is varied on the $\mathrm{x}$-axis.

As a whole, the K2 group results had larger dispersion compared to K3 groups indicated by larger IQR values at all speeds. Moreover, the error distribution was highly skewed for K2 subjects as indicated by asymmetrical boxplots. On the other hand, walking speed had a significant effect on the TO prediction error on the sound side only for K3 subjects (pi.05, Friedman test). Other variables did not exhibit any significant correlation with walking speed. 

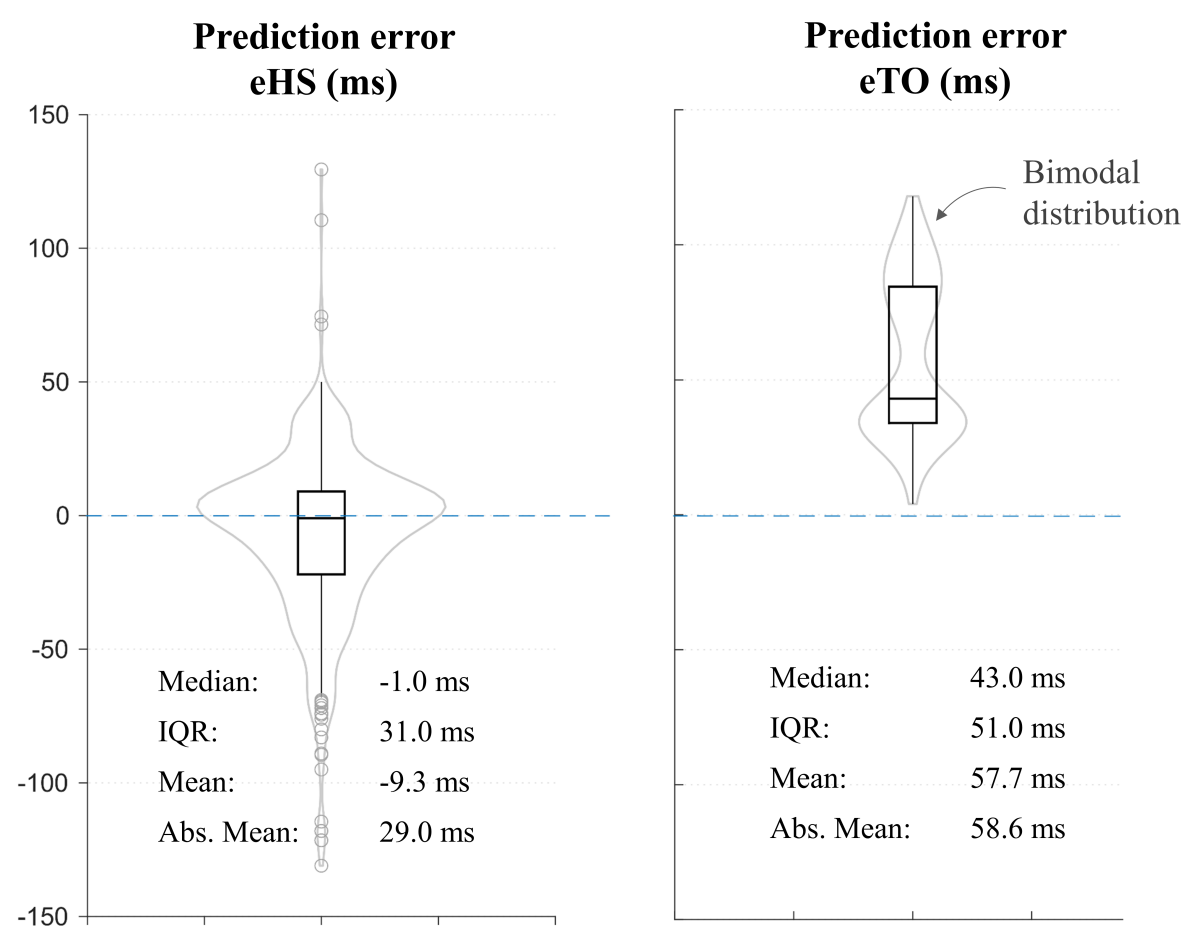

Figure 2: Heel strike (left) and Toe-off (right) error plots for all 2595 walking cycles. Boxplots (black) are superimposed with violin plots (in grey) indicating error distribution. Key statistics are also indicated below each plot. The box indicates the lower and upper quartiles with the central line showing the median. The top and bottom lines of the box represent, respectively, the medians for the upper and lower halves of the data and the whiskers represent the highest and lowest values of the distribution, excluding outliers. Outliers are also presented as circles

\section{Discussion and conclusion}

This is one of the most comprehensive studies for any population which compares kinematics-based TO and HS prediction against the force platform data. The results provide an insight into the degree of credence of velocity-based gait event prediction. At the same time, the results can be exploited by researchers for event prediction in the absence of force platforms.

A key finding from this study is the consistent early prediction of the toe-off event for both legs. This means that the actual toe-off takes place after the $1^{\text {st }}$ negative peak or minima of the velocity signal and points towards an inherent limitation of this algorithm. In physiological terms, it indicates that the leg has already started to accelerate forward before the foot leaves the ground. Some authors have proposed the point of zerocrossing (the point where the signal crosses the from negative-to-positive) as TO event (e.g. [27]). However, observation of our velocity signals for numerous trials does not support this view. We postulate that the actual TO event occurs after the negative peak (NP) but before the zero-crossing 


\section{Prediction error eHS (ms)}

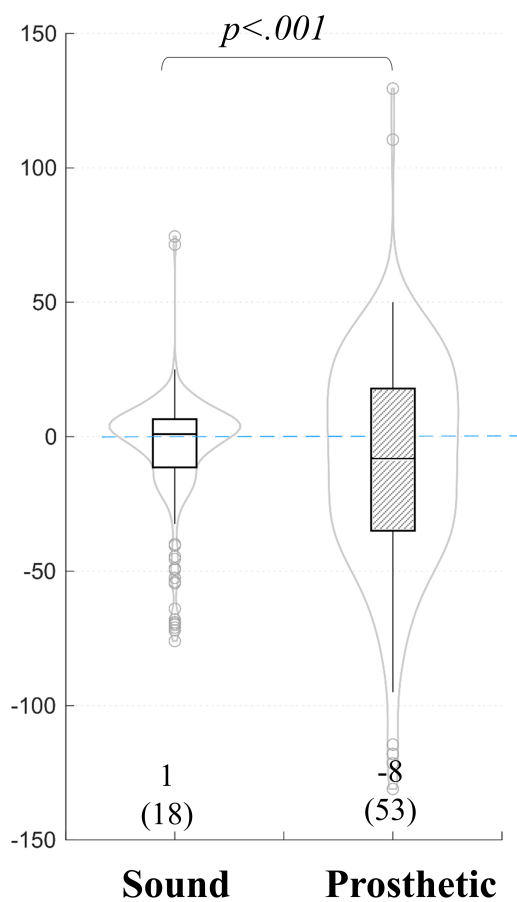

\section{Prediction error eTO (ms)}

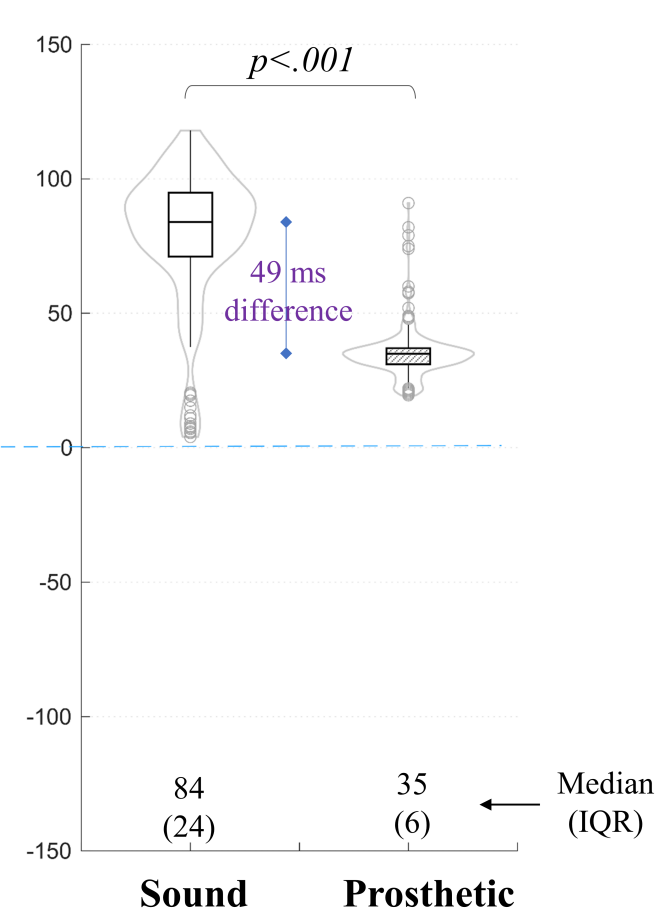

Figure 3: Separate boxplots and error distribution for sound and prosthetic legs. Median and interquartile ranges are mentioned at the bottom in each case. Significant differences were found between errors on the sound and prosthetic sides.

(ZC) of the velocity signal, hence yielding a narrow NP-ZC zone. Future studies should focus on this zone for accurate prediction of TO event.

On the other hand, the HS prediction stretched both positive and negative values, indicating early and late prediction respectively. The error magnitude was however smaller indicating that the actual heel-strike occurs in a small window around the $2^{\text {nd }}$ negative peak. The result of this study can be used to define a search window around the negative peaks of the leg velocity graph as shown in Figure 5. For the HS, the window stretches about $50 \mathrm{~ms}$ in both directions of the first negative peak. Similarly, for the TO, the actual event window stretches up to $100 \mathrm{~ms}$ after the second negative peak. Future research should focus on these windows while improving the prediction accuracy of kinematics-based methods possibly by including information from other signals such as foot and/or leg accelerations.

\section{Leg and group effects}

Group comparisons indicated that the eTO prediction accuracy was better on the prosthetic side (Figure 3 ) and further correlated negatively with walking speed (Figure 4) on the sound side. On the other hand, the 


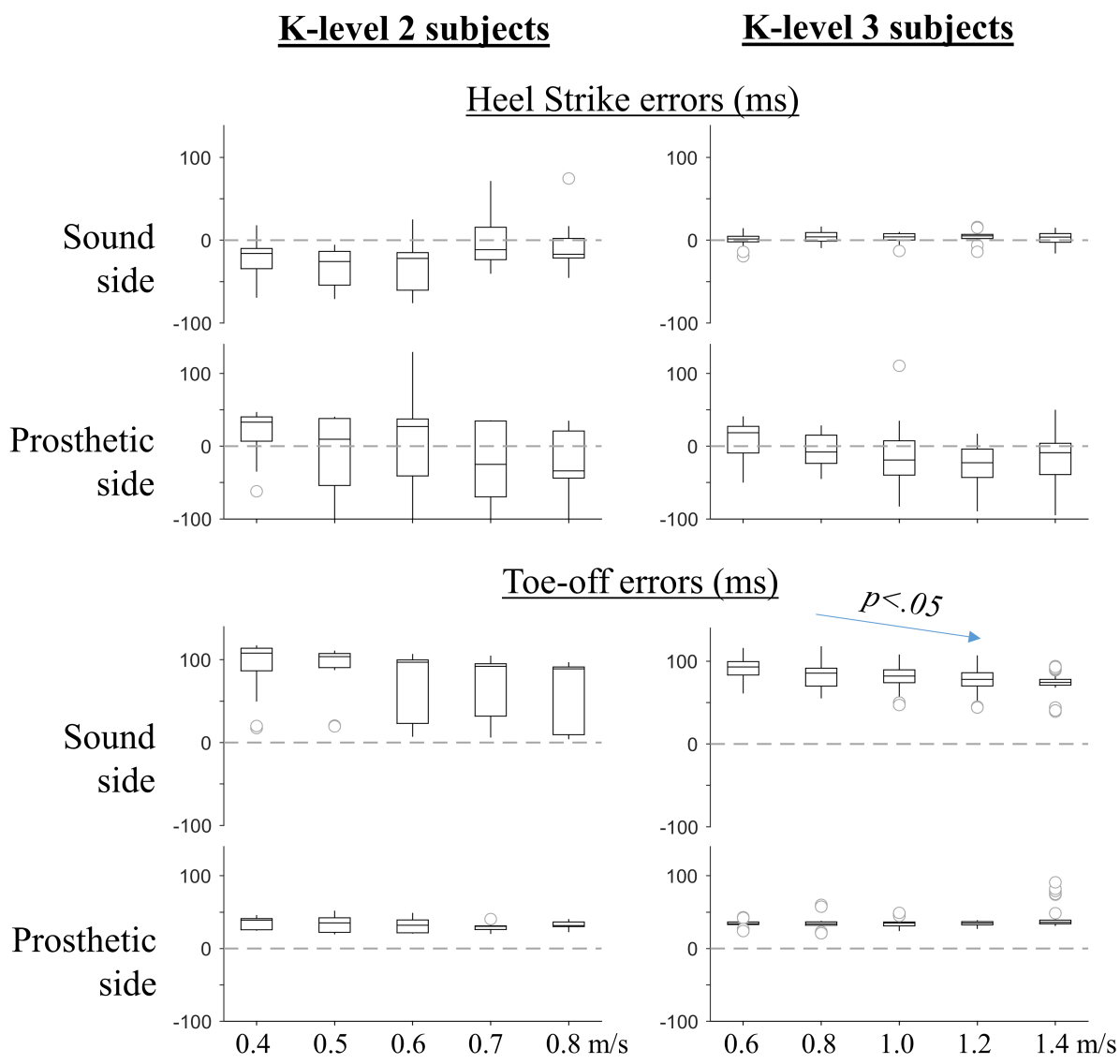

Figure 4: Toe-off (top panel) and Heel-strike (bottom) median error values separated by subject groups (K-level 2 on left and K-level 3 on right) and leg side. Speed is varied on $\mathrm{x}$-axis for each group $(0.4$ to $0.8 \mathrm{~m} / \mathrm{s}$ for $\mathrm{K} 2$ subjects, 0.6 to $1.4 \mathrm{~m} / \mathrm{s}$ for $\mathrm{K} 3$ subjects)

eHS variability was larger for K-level 2 subject group which warrants precaution when using the kinematics method for subjects classified as 'limited community ambulators' on the functional classification system.

\section{Comparison with literature studies}

As mentioned in the beginning, there are barely any published studies with the amputee population which makes a direct comparison of results difficult. Nevertheless, a comparison of the results of this study with the available literature is presented in Table 2. Almost all studies have reported early TO prediction with this algorithm, albeit with smaller magnitudes than our results. Catalfamo et. al. [2] reported early TO prediction for all steps with a mean error of 50-73ms for healthy and children suffering from cerebral palsy. Trojaniello et. al. [14] reported mean absolute TO errors in the range of 16 to $22 \mathrm{~ms}$ for elderly and gait-impaired subjects. These smaller magnitudes further reinforce our premise that the actual TO event occurs in the NP-ZC zone mentioned earlier for all populations. 


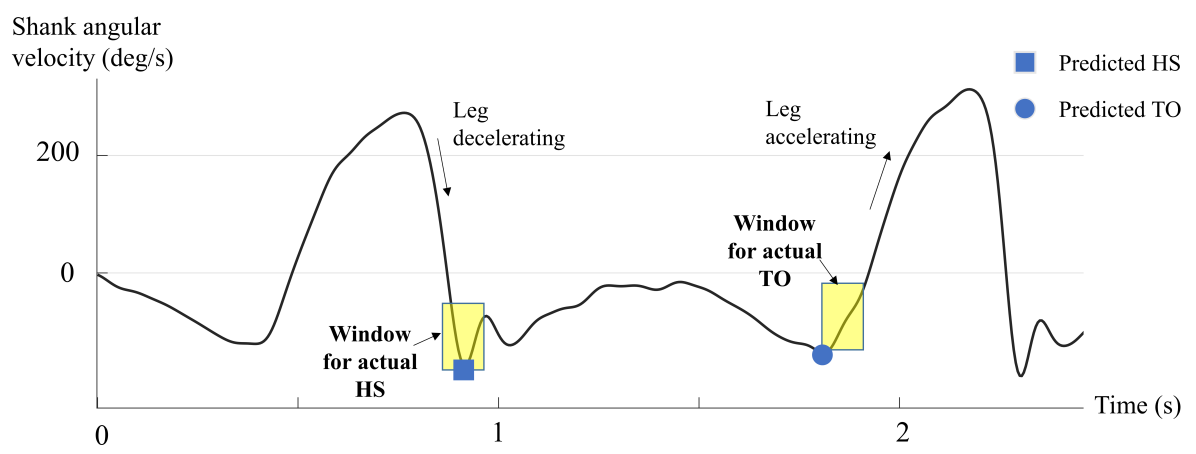

Figure 5: Typical shank velocity graph supplemented with the windows likely to contain the HS and TO events for a gait cycle based on the results of this study.

Similarly, for HS prediction, the error values are smaller than for TO prediction as in this study. For instance, Zahradka et al. [19] reported a mean error of $-10.45 \mathrm{~ms}$ for a group of healthy and gait-impaired subjects which is very close to our results. Storm et. al. [3] reported absolute mean error for indoor and outdoor walking in healthy adults in the range of $11-14 \mathrm{~ms}$. The study by Catalfamo et. al [2] reported a mean HS error of $-8 \mathrm{~ms}$, an absolute mean of $15 \mathrm{~ms}$ for level-ground walking. All in all, the findings of this study match well with the literature and indicate a higher level of confidence for the HS prediction than for the TO prediction.

In conclusion, it is possible to detect heel-strike and toe-off events for the amputee population using the leg velocity, albeit less accurately for the toe-off event on the sound side and for patients with limited community ambulation ability.

\section{References}

[1] Jacquelin Perry and Judith M. Burnfield. Gait Analysis: Normal and Pathological Function. Slack Thorofare, NJ, 1992.

[2] Paola Catalfamo, Salim Ghoussayni, and David Ewins. Gait event detection on level ground and incline walking using a rate gyroscope. Sensors, 10(6):5683-5702, 2010.

[3] Fabio A Storm, Christopher J Buckley, and Claudia Mazzà. Gait event detection in laboratory and real life settings: Accuracy of ankle and waist sensor based methods. Gait $\mathscr{E}$ posture, 50:42-46, 2016.

[4] Darwin Gouwanda and Alpha Agape Gopalai. A robustreal-time gaiteventdetection using wirelessgyroscope and itsapplication on normal and alteredgaits. Medical Engineering and Physics, 37(2):219$225,2015$.

[5] Shuo Ding, Xiaoping Ouyang, Tao Liu, Zhihao Li, and Huayong Yang. Gait event detection of a lower extremity exoskeleton robot by an intelligent IMU. IEEE Sensors Journal, 18(23):9728-9735, 2018. 
[6] Hongyu Zhao, Zhelong Wang, Sen Qiu, Jiaxin Wang, Fang Xu, Zhengyu Wang, and Yanming Shen. Adaptive gait detection based on foot-mounted inertial sensors and multi-sensor fusion. Information Fusion, 52(November 2018):157-166, 2019.

[7] Cagla Fadillioglu, Bernd J. Stetter, Steffen Ringhof, Frieder C. Krafft, Stefan Sell, and Thorsten Stein. Automated gait event detection for a variety of locomotion tasks using a novel gyroscope-based algorithm. Gait and Posture, 81:102-108, 2020.

[8] S. Sahoo, M. Saboo, D. K. Pratihar, and S. Mukhopadhyay. RealTime Detection of Actual and Early Gait Events During LevelGround and Ramp Walking. IEEE Sensors, 20(14):8128-8136, 2020.

[9] A. R. De Asha, M. A. Robinson, and G. J. Barton. A marker based kinematic method of identifying initial contact during gait suitable for use in real-time visual feedback applications. Gait and Posture, 36(3):650-652, 2012.

[10] Jan M Jasiewicz, John H J Allum, James W Middleton, Andrew Barriskill, Peter Condie, Brendan Purcell, and Raymond Che Tin Li. Gait event detection using linear accelerometers or angular velocity transducers in able-bodied and spinal-cord injured individuals. Gait Es posture, 24(4):502-509, 2006.

[11] Ciara M. O'Connor, Susannah K. Thorpe, Mark J. O'Malley, and Christopher L. Vaughan. Automatic detection of gait events using kinematic data. Gait and Posture, 25(3):469-474, 2007.

[12] Rejane Vale Gonçalves, Sérgio Teixeira Fonseca, Priscila Albuquerque Araújo, Vanessa Lara Araújo, Tais Martins Barboza, Gabriela Andrade Martins, and Marisa Cotta Mancini. Identification of gait events in children with spastic cerebral palsy: comparison between the force plate and algorithms. Brazilian Journal of Physical Therapy, 24(5):392-398, 2020.

[13] Ahad Behboodi, Nicole Zahradka, Henry Wright, James Alesi, and Samuel C.K. Lee. Real-time detection of seven phases of gait in children with cerebral palsy using two gyroscopes. Sensors (Switzerland), 19(11):7-11, 2019.

[14] Diana Trojaniello, Andrea Cereatti, Elisa Pelosin, Laura Avanzino, Anat Mirelman, Jeffrey M. Hausdorff, and Ugo Della Croce. Estimation of step-by-step spatio-temporal parameters of normal and impaired gait using shank-mounted magneto-inertial sensors: Application to elderly, hemiparetic, parkinsonian and choreic gait. Journal of NeuroEngineering and Rehabilitation, 11(1):152, 2014.

[15] Rosa M.S. Visscher, Sailee Sansgiri, Marie Freslier, Jaap Harlaar, Reinald Brunner, William R. Taylor, and Navrag B. Singh. Towards validation and standardization of automatic gait event identification algorithms for use in paediatric pathological populations. Gait and Posture, 86(March):64-69, 2021.

[16] Sandra R. Hundza, William R. Hook, Christopher R. Harris, Sunny V. Mahajan, Paul A. Leslie, Carl A. Spani, Leonhard G. Spalteholz, Benjamin J. Birch, Drew T. Commandeur, and Nigel J. Livingston. Accurate and reliable gait cycle detection in parkinson's 
disease. IEEE Transactions on Neural Systems and Rehabilitation Engineering, 22(1):127-137, 2014.

[17] Kamiar Aminian, B Najafi, C Büla, P-F Leyvraz, and Ph Robert. Spatio-temporal parameters of gait measured by an ambulatory system using miniature gyroscopes. Journal of biomechanics, 35(5):689699, 2002 .

[18] Wesley Niswander and Kimberly Kontson. Evaluating the impact of imu sensor location and walking task on accuracy of gait event detection algorithms. Sensors, 21(12), 2021.

[19] Nicole Zahradka, Khushboo Verma, Ahad Behboodi, Barry Bodt, Henry Wright, and Samuel C.K. Lee. An evaluation of three kinematic methods for gait event detection compared to the kinetic-based 'gold standard'. Sensors (Switzerland), 20(18):1-15, 2020.

[20] Hafiz Farhan Maqbool, Muhammad Afif B Husman, Mohammed I Awad, Alireza Abouhossein, and Abbas A Dehghani-Sanij. Realtime gait event detection for transfemoral amputees during ramp ascending and descending. In Engineering in Medicine and Biology Society (EMBC), 37th Annual International Conference of the IEEE, pages 4785-4788. IEEE, 2015.

[21] Paolo Fraccaro, Lorcan Walsh, Julie Doyle, and Dympna O'Sullivan. Real-world Gyroscope-based Gait Event Detection and Gait Feature Extraction. In eTELEMED 2014, The Sixth International Conference on eHealth, Telemedicine, and Social Medicine, number c, pages $247-252,2014$.

[22] Jung Keun Lee and Edward J Park. Quasi real-time gait event detection using shank-attached gyroscopes. Medical and Biological Engineering and Computing, 49(6):707-712, 2011.

[23] Sarah Hood, Marshall K. Ishmael, Andrew Gunnell, K. B. Foreman, and Tommaso Lenzi. A kinematic and kinetic dataset of 18 aboveknee amputees walking at various speeds. Scientific Data, 7(1):1-8, 2020 .

[24] Robert S. Gailey, Kathryn E. Roach, E.Brooks Applegate, Brandon Cho, Bridgid Cunniffe, Stephanie Licht, Melanie Maguire, and Mark S. Nash. The Amputee Mobility Predictor: An instrument to assess determinants of the lower-limb amputee's ability to ambulate. Archives of Physical Medicine and Rehabilitation, 83(5):613627, 2002.

[25] Arnaud Barre and Stéphane Armand. Biomechanical ToolKit: Opensource framework to visualize and process biomechanical data. Computer Methods and Programs in Biomedicine, 114(1):80-87, 2014.

[26] David A. Winter. Biomechanics and Motor Control of Human Movement: Fourth Edition. John Wiley \& Sons, 2009.

[27] E. Allseits, J. Lučarević, R. Gailey, V. Agrawal, I. Gaunaurd, and C. Bennett. The development and concurrent validity of a realtime algorithm for temporal gait analysis using inertial measurement units. Journal of Biomechanics, 55:27-33, apr 2017. 
Table 2: A comparison with the error magnitudes found in this study and the available relevant literature, ME: Mean Error, MAE: Mean Absolute Error

\begin{tabular}{|c|c|c|c|c|}
\hline Study & $\begin{array}{l}\text { Subject pop- } \\
\text { ulation and } \\
\text { task }\end{array}$ & $\begin{array}{l}\text { Prediction } \\
\text { method }\end{array}$ & HS error (ms) & TO error (ms) \\
\hline This study & $\begin{array}{l}\text { Amputee, } \\
\mathrm{N}=10 \text {, Level } \\
\text { treadmill } \\
\text { walking }\end{array}$ & $\begin{array}{l}\text { Leg kinematics, } \\
\text { Dual-minima of } \\
\text { shank velocity }\end{array}$ & $\begin{array}{l}\text { ME: -9.3, MAE: } \\
29\end{array}$ & $\begin{array}{l}\text { ME: } \quad 57.7, \\
\text { MAE: } 58.6\end{array}$ \\
\hline $\begin{array}{l}\text { Maqbool et. } \\
\text { al. } 2015 \text { [20] }\end{array}$ & $\begin{array}{l}\text { Amputee, } \\
\mathrm{N}=1, \quad \text { Ramp } \\
\text { ascent } \\
\text { descent }\end{array}$ & $\begin{array}{l}\text { Leg kinematics, } \\
\text { Dual-minima }\end{array}$ & ME: -37 to 13 & ME: -17 to 122 \\
\hline $\begin{array}{l}\text { Storm et al. } \\
2016[3]\end{array}$ & $\begin{array}{l}\text { Healthy, } \\
\mathrm{N}=10 \text {, Over- } \\
\text { ground }\end{array}$ & $\begin{array}{l}\text { Minima of shank } \\
\text { velocity for HS. } \\
\text { Acceleration- } \\
\text { based for TO }\end{array}$ & MAE: 7 to 14 & MAE: 16 to 51 \\
\hline $\begin{array}{l}\text { Zahradka et } \\
\text { al. } 2020 \text { [19] }\end{array}$ & $\begin{array}{l}\text { Healthy } \\
\text { and Gait- } \\
\text { impaired, } \\
\mathrm{N}=17, \text { Level } \\
\text { treadmill } \\
\text { walking }\end{array}$ & $\begin{array}{l}\text { Minima of shank } \\
\text { velocity for TO. } \\
\text { Zero-crossing for } \\
\text { HS }\end{array}$ & ME: -10.45 & ME: -56.20 \\
\hline $\begin{array}{l}\text { Trojaniello } \\
\text { et. al. } 2014 \\
\text { [14] }\end{array}$ & $\begin{array}{l}\text { Healthy } \\
\text { and Gait- } \\
\text { impaired, } \\
\mathrm{N}=40, \text { Over- } \\
\text { ground }\end{array}$ & $\begin{array}{l}\text { Minima of shank } \\
\text { velocity for HS. } \\
\text { Acceleration- } \\
\text { based for TO }\end{array}$ & $\begin{array}{l}\text { ME: } 0 \text { to }-22, \\
\text { MAE } 10 \text { to } 22\end{array}$ & $\begin{array}{l}\text { ME: } 0 \text { to }-16 \text {, } \\
\text { MAE: } 16 \text { to } 22\end{array}$ \\
\hline $\begin{array}{l}\text { Lee \& Park } \\
2011[22]\end{array}$ & $\begin{array}{l}\text { Healthy, } \mathrm{N}=5 \text {, } \\
\text { Overground }\end{array}$ & $\begin{array}{l}\text { Leg kinematics, } \\
\text { Dual-minima }\end{array}$ & ME: -17 to -21 & ME: 3 to 15 \\
\hline $\begin{array}{l}\text { Catalfamo } \\
\text { et. al. } 2010 \\
{[2]}\end{array}$ & $\begin{array}{l}\text { Healthy and } \\
\text { CP, } \quad \mathrm{N}=7 \text {, } \\
\text { Overground } \\
\text { and ramp }\end{array}$ & $\begin{array}{l}\text { Leg kinematics, } \\
\text { Dual-minima }\end{array}$ & $\begin{array}{l}\text { ME: }-8 \text { to }-21 \text {, } \\
\text { MAE: } 15 \text { to } 24\end{array}$ & $\begin{array}{l}\text { ME: } 50 \text { to } 73 \text {, } \\
\text { MAE: } 50 \text { to } 73\end{array}$ \\
\hline
\end{tabular}


Prediction error eHS (ms)

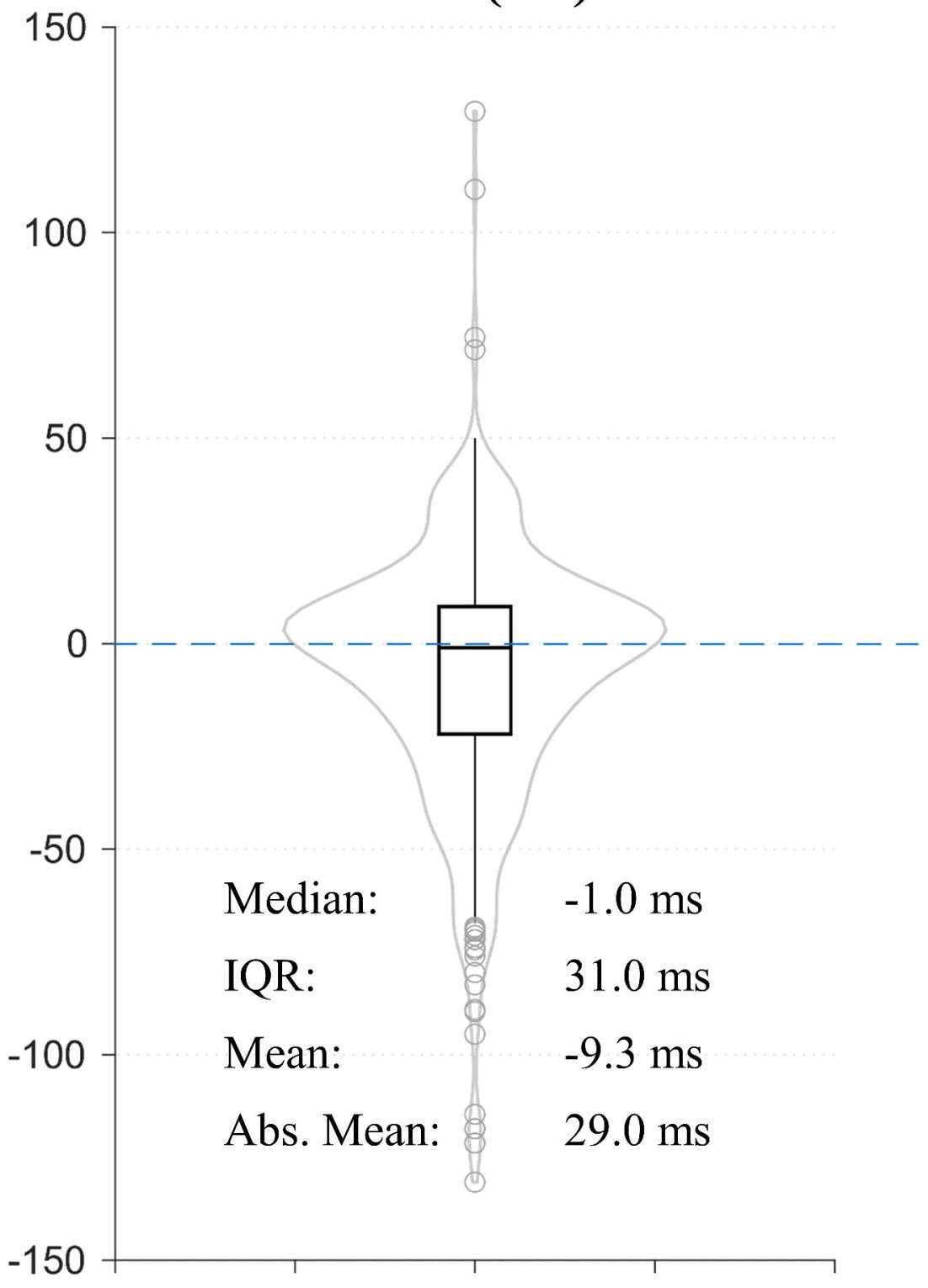

Prediction error eTO (ms)

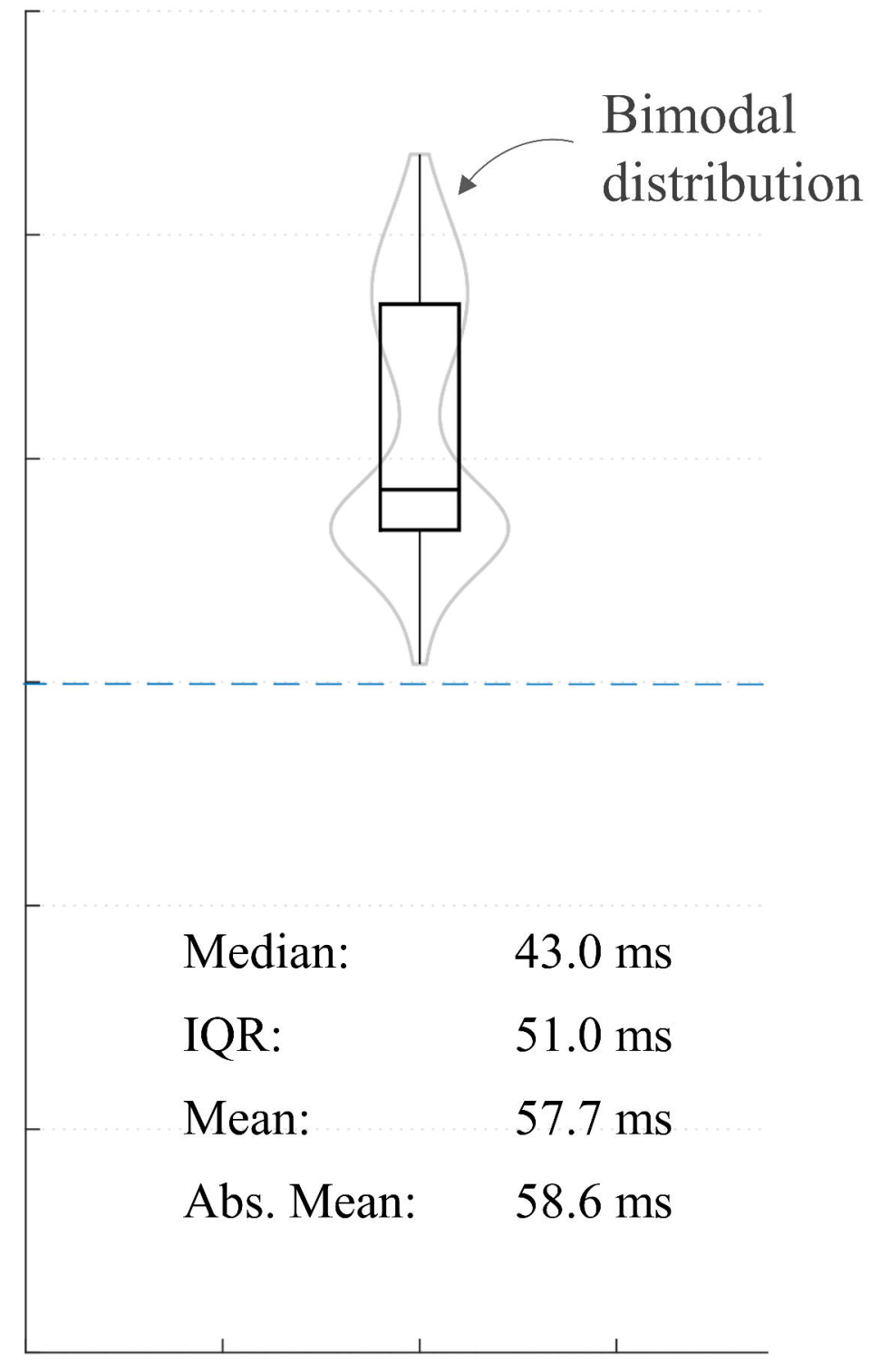




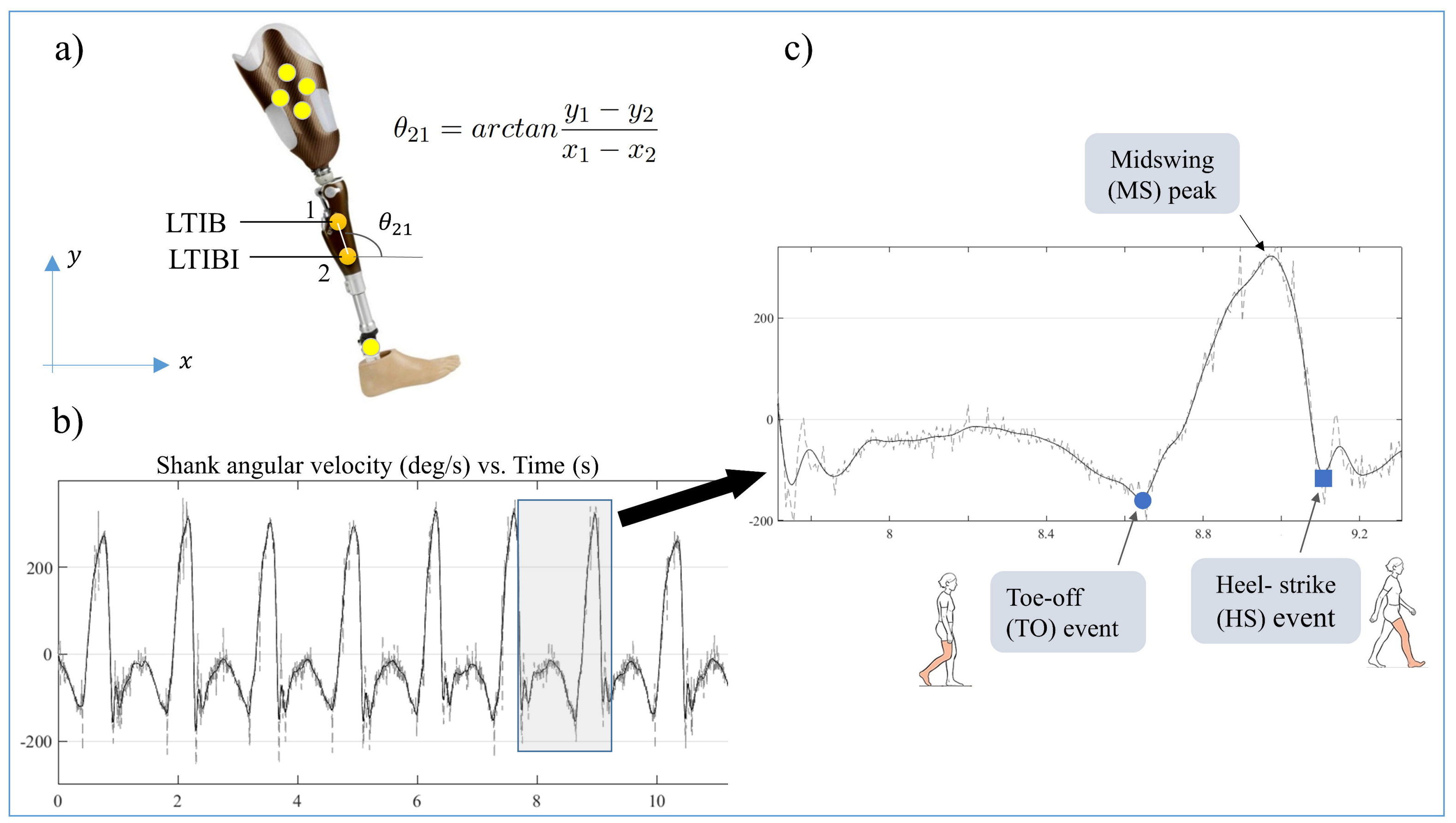


Heel Strike errors (ms)
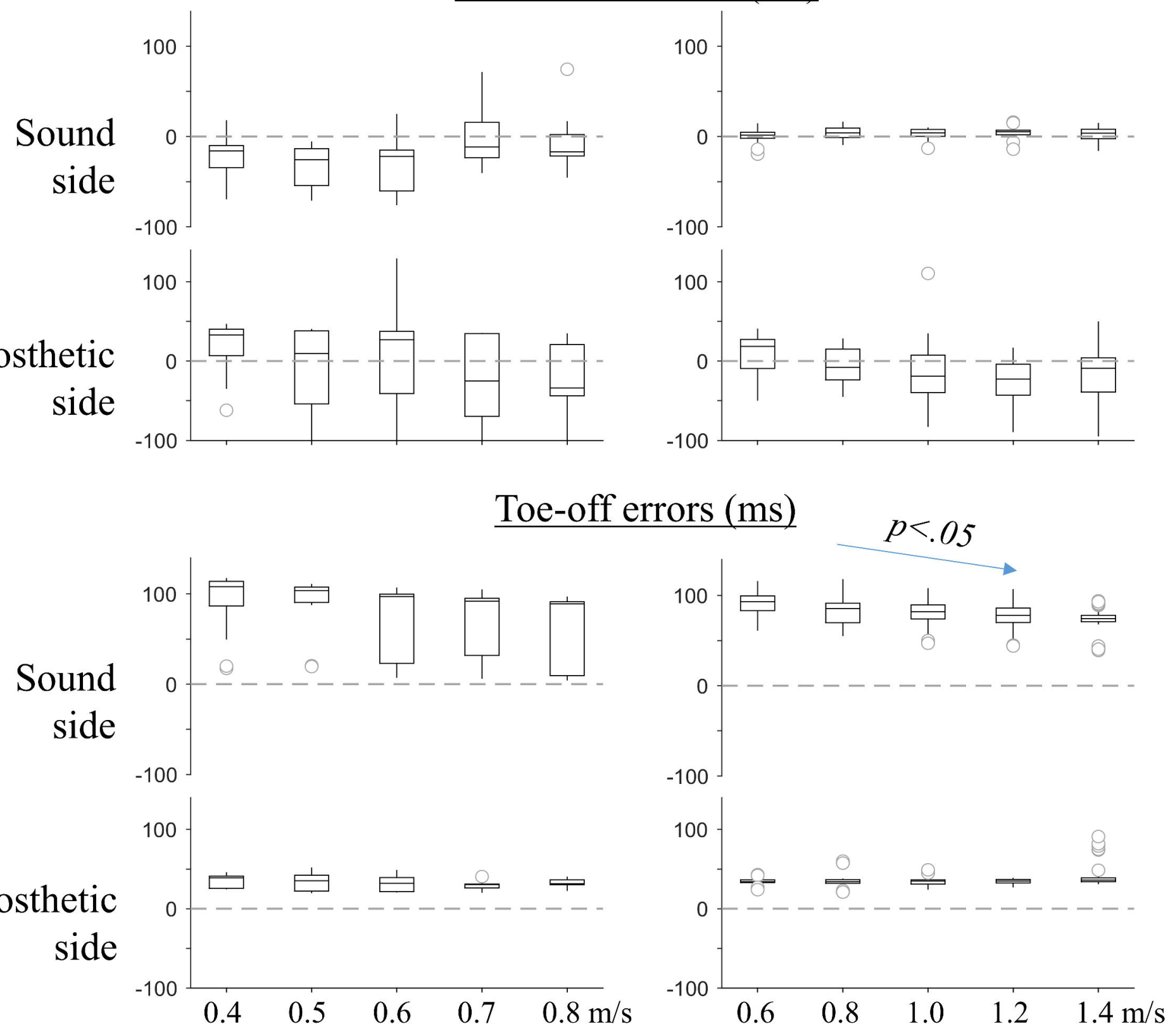
Prediction error eHS (ms)

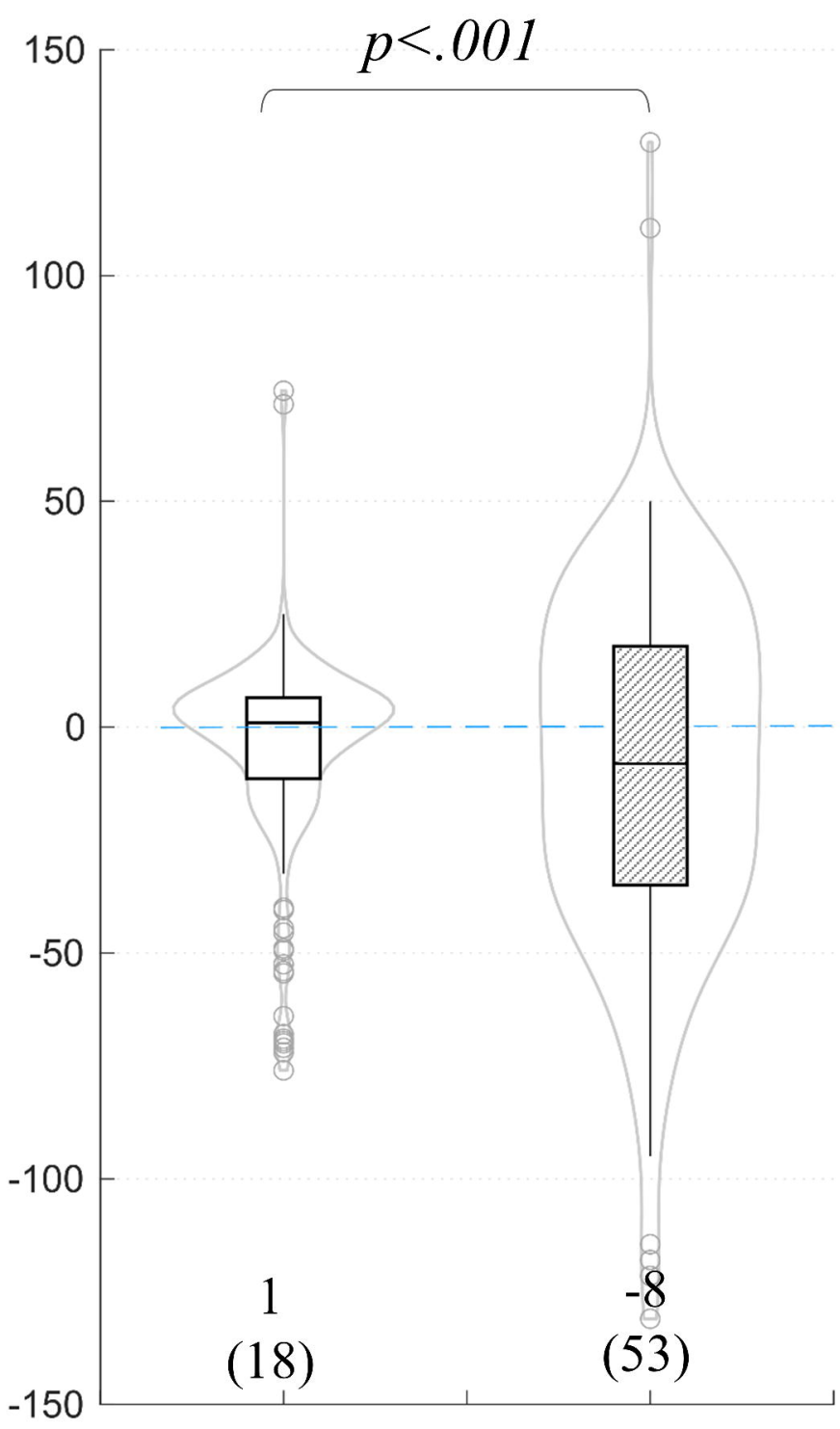

Sound
Prediction error eTO (ms)

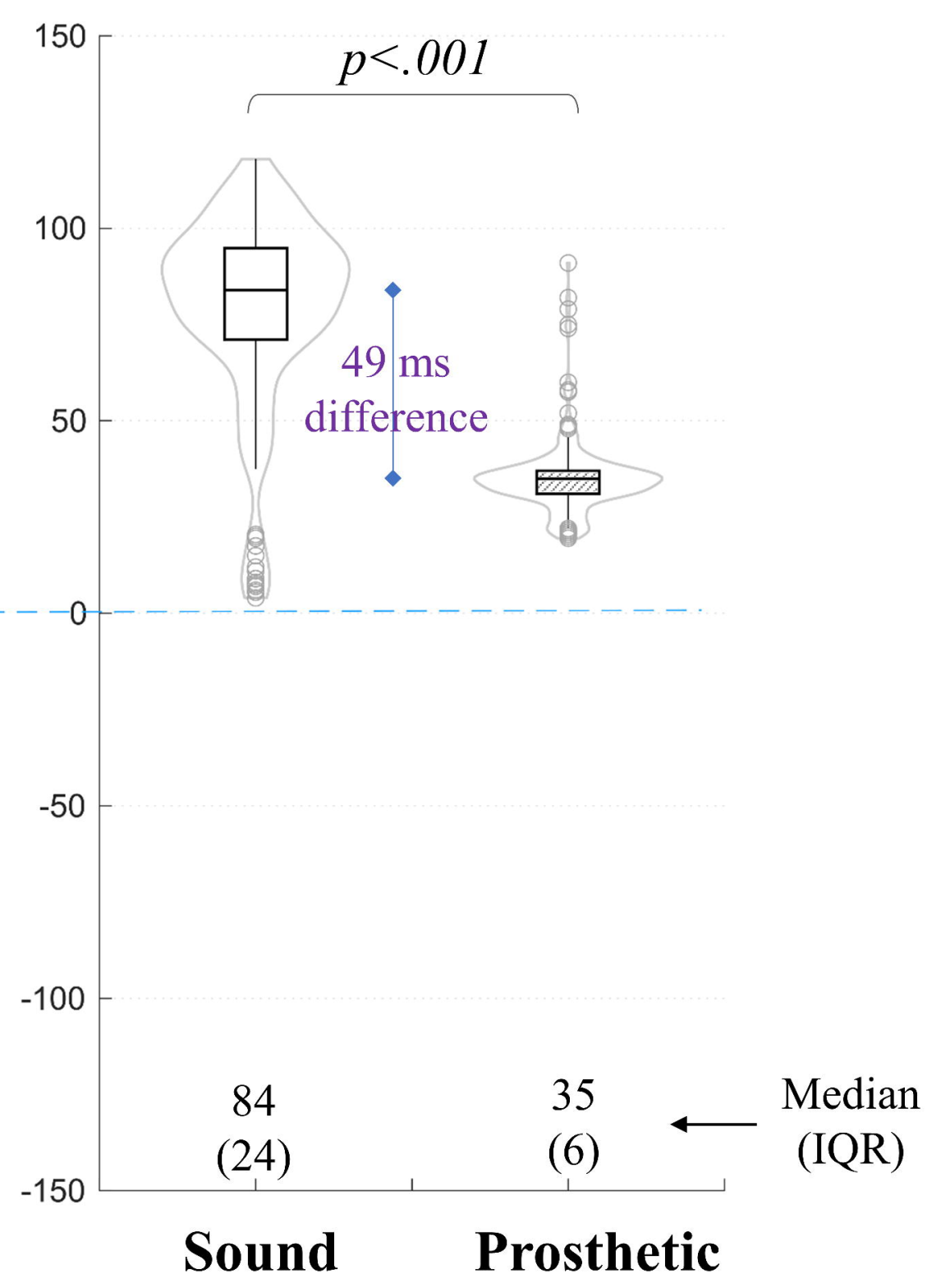


